\title{
Learning-Related Facilitation of Rhinal Interactions by Medial Prefrontal Inputs
}

\author{
Rony Paz, Elizabeth P. Bauer, and Denis Paré \\ Center for Molecular and Behavioral Neuroscience, Rutgers State University, Newark, New Jersey 07102
}

\begin{abstract}
Much data suggests that hippocampal-medial prefrontal cortex (mPFC) interactions support memory consolidation. This process is thought to involve the gradual transfer of transient hippocampal-dependent memories to distributed neocortical sites for long-term storage. However, hippocampal projections to the neocortex involve a multisynaptic pathway that sequentially progresses through the entorhinal and perirhinal regions before reaching the neocortex. Similarly, the mPFC influences the hippocampus via the rhinal cortices, suggesting that the rhinal cortices occupy a strategic position in this network. The present study thus tested the idea that the mPFC supports memory by facilitating the transfer of hippocampal activity to the neocortex via an enhancement of entorhinal to perirhinal communication. To this end, we simultaneously recorded mPFC, perirhinal, and entorhinal neurons during the acquisition of a traceconditioning task in which a visual conditioned stimulus (CS) was followed by a delay period after which a liquid reward was administered. At learning onset, correlated perirhinal-entorhinal firing increased in relation to mPFC activity, but with no preferential directionality, and only after reward delivery. However, as learning progressed across days, mPFC activity gradually enhanced rhinal correlations in relation to the CS as well, and did so in a specific direction: from entorhinal to perirhinal neurons. This suggests that, at late stages of learning, $\mathrm{mPFC}$ activity facilitates entorhinal to perirhinal communication. Because this connection is a necessary step for the transfer of hippocampal activity to the neocortex, our results suggest that the mPFC is involved in the slow iterative process supporting the integration of hippocampal-dependent memories into neocortical networks.
\end{abstract}

Key words: entorhinal; extracellular recording; hippocampus; memory; prefrontal; reward

\section{Introduction}

The hippocampus plays a time-limited role in the formation of declarative memories, with memories gradually becoming independent of the hippocampus over time (Zola-Morgan and Squire, 1986; Kim and Fanselow, 1992). It is believed that these remote memories are gradually transferred from the hippocampus to the neocortex for long-term storage (Wiltgen et al., 2004). In keeping with this, the hippocampus and neocortex show inverse gradients of activity in relation to the retrieval of recent versus remote spatial memories (Bontempi et al., 1999). In particular, remote memories produce stronger activations in temporal and frontal neocortical areas than recent ones and inversely in the hippocampus (Bontempi et al., 1999; Takashima et al., 2006).

A related line of investigation indicates that the medial prefrontal cortex (mPFC) is critical for the consolidation of hippocampal-dependent memories. In trace-conditioning tasks for instance, hippocampal lesions cause a severe deficit when made soon after training, but not after a month, whereas mPFC lesions produce the opposite pattern of impairments (Takehara et al., 2003). Consistent with this, expression of the immediate

Received March 9, 2007; revised May 4, 2007; accepted May 7, 2007

This work was supported by the National Science Foundation under Grant 0208712 and by National Institute of Mental Health Grant R01 MH073610.

Correspondence should be addressed to Denis Paré, Center for Molecular and Behavioral Neuroscience, Rutgers,

The State University of New Jersey, 197 University Avenue, Newark, NJ 07102. E-mail: pare@axon.rutgers.edu. DOI:10.1523/JNEUROSCI.1077-07.2007

Copyright $\odot 2007$ Society for Neuroscience $\quad$ 0270-6474/07/276542-10\$15.00/0 early gene zif268 is enhanced in the MPFC by remote, but not recent, contextual memory tests (Frankland et al., 2004). Moreover, local intra-mPFC injections of NMDA antagonists within 2 weeks after training impair long-term recall, implicating the mPFC in memory consolidation (Takehara-Nishiuchi et al., 2006). Last, functional imaging studies in humans have revealed that as the retention interval increases, memory retrieval produces gradually stronger activations in the mPFC whereas the opposite is seen in the hippocampus (Takashima et al., 2006).

Overall, this data suggests that in the first few weeks after training, memories become gradually independent of the hippocampus and that, in parallel, they progressively become more dependent on mPFC activity.

Although hippocampal projections to the mPFC (Swanson, 1981; Ferino et al., 1987; Jay and Witter, 1991; Thierry et al., 2000) likely support mPFC involvement in memory, the role of $\mathrm{mPFC}$ activity in memory formation remains unclear. One possibility is that $\mathrm{mPFC}$ affects the transfer of hippocampal activity toward the neocortex. Consistent with this possibility, the mPFC projects to the rhinal cortices (Room et al., 1985; Sesack et al., 1989; Hurley et al., 1991), the main route for impulse traffic into and out of the hippocampus. Indeed, most hippocampal projections to the neocortex involve a multisynaptic pathway that sequentially progresses through the entorhinal (ER) cortex, perirhinal (PR) areas 35 and 36, and then the neocortex. Moreover, pretraining ER lesions impair acquisition on a traceconditioning task (Ryou et al., 2001) and multiple lines of evi- 
dence implicate the rhinal cortices in memory formation (Meunier et al., 1993; Suzuki and Eichenbaum, 2000; Muir and Bilkey, 2003; Murray et al., 2005; Leutgeb et al., 2005). Thus, the present study was undertaken to test the idea that the mPFC influences memory formation by modulating interactions between the neocortex and hippocampus at the level of the rhinal cortices. To this end, we examined the relative timing of unit activity in the $\mathrm{MPFC}, \mathrm{PR}$, and ER cortices during the acquisition of a trace-conditioning task.

\section{Materials and Methods \\ Surgery}

Three adult cats were implanted with two arrays of high-impedance microelectrodes aimed at the mPFC (see Fig. 1A1) and the rhinal cortices (see Fig. 1 A2). All procedures were approved by the Institutional Animal Care and Use Committee of Rutgers University, in compliance with the Guide for the Care and Use of Laboratory Animals (Department of Health and Human Services).

Cats were preanesthetized with ketamine and xylazine (15 and $2 \mathrm{mg} /$ $\mathrm{kg}$, i.m.) and artificially ventilated with a mixture of ambient air, oxygen, and isoflurane. Atropine $(0.05 \mathrm{mg} / \mathrm{kg}$, i.m. $)$ was administered. The endtidal $\mathrm{CO}_{2}$ concentration was maintained at $3.7 \pm 0.2 \%$, and the body temperature at $37-38^{\circ} \mathrm{C}$ with a heating pad. Bupivacaine (subcutaneous) was administered in the region to be incised $15 \mathrm{~min}$ before the first incision. In sterile conditions, an incision was performed on the midline of the scalp and the skull muscles were retracted. After trepanation and opening of the dura mater, an array of microelectrodes was stereotaxically lowered until the electrodes reached the deep rhinal layers. A second array was inserted in the $\mathrm{mPFC}$ with an oblique, lateromedial approach (Fig. $1 B$ ).

Finally, four screws were cemented to the skull to later fix the cat's head without pain or pressure. At the end of the surgery, the animals were administered penicillin (20,000 UI/kg, i.m.) and an analgesic (Ketophen, $2 \mathrm{mg} / \mathrm{kg}$, s.c., daily for $3 \mathrm{~d}$ ). Recording sessions began $8 \mathrm{~d}$ after the surgery.

\section{Behavior}

The learning task was an appetitive trace-conditioning paradigm in which a visual CS $(1.5 \mathrm{~s})$ was followed by a $1.5 \mathrm{~s}$ delay period, after which a liquid reward was administered. Trials occurred at random intervals of $30-90 \mathrm{~s}$. The animals performed 38-95 trials (median 55.5) per daily session for 9 consecutive days. The liquid reward $(2 \mathrm{ml} /$ trial $)$ was a preferred food [Gerber's (Fremont, MI) pureed baby food "sweet potatoes and turkey"]. The animals were only fed during recording sessions, and their weight was monitored daily to maintain body weight within $10 \%$ of its initial value.

Behavior was monitored by means of a switch detecting when the tongue of the animals contacted the receptacle where the food reward was administered. The visual CS was a global change in the illumination (from black to white) of a 12 inch liquid crystal display (LCD) screen placed one foot in from of the animals. Detection of the visual CS did not necessitate that the animals maintain a fixed gaze at the center of the LCD screen because it encompassed most of their visual field. Because the animals were hungry, they were highly aroused and remained awake at all times (as assessed by EEG recordings) with their eyes opened.

\section{Histology}

At the end of the experiments, the animals were given an overdose of sodium pentobarbital $(50 \mathrm{mg} / \mathrm{kg}$, i.v. ) and perfused fixed. The recording sites were marked with electrolytic lesions $(0.5 \mathrm{~mA}, 5 \mathrm{~s})$ (see Fig. $1 B$ ). The brains were later sectioned on a vibrating microtome (at $100 \mu \mathrm{m}$ ) and stained with cresyl violet to verify the position of recording electrodes. Microelectrode tracks were reconstructed by combining micrometer readings with the histology. This report includes only neurons that were histologically confirmed to be located in the regions of interest.

\section{Recording and analysis methods}

Neuronal activity was sampled at $100 \mu \mathrm{m}$ intervals. Each time the electrodes were moved to a new site (once a day), 30 min elapsed before data were acquired to ensure mechanical stability. The signals picked up by the electrodes (Fig. 1C) were observed on an oscilloscope, digitized, and stored on a hard disk. Spike sorting was performed off-line using a clustering algorithm based on principal component analysis and $K$-means. Computation of spike-triggered joint histograms. To analyze the relative timing of mPFC, PR, and ER activity, we computed spike-triggered joint histograms (STJHs), as described in Results. The STJH is an adaptation of the joint peristimulus time histogram (JPSTH) method (Aertsen et al., 1989) where, instead of external stimuli, mPFC spikes are used as a temporal reference to study correlated PR and ER firing. Thus, like JPSTHs, STJHs are "trial-based," only instead of stimuli, as in the standard JPSTHs, our STJHs were mPFC-spike-based. The STJH is computed by taking $\pm 150 \mathrm{~ms}$ segments of rhinal activity around mPFC spikes (time 0 ), and by plotting the spikes of the PR cell on the $x$-axis and of the ER cell on the $y$-axis. The bins of the STJH that contain a coincidence of spikes are incremented. Repeating this process for each mPFC spike gradually produces the raw STJH. For presentation purposes only, the STJHs were smoothed using a two-dimensional Gaussian with variance equal to $25 \mathrm{~ms}$.

Statistical significance of STJHs. This was assessed by performing bin by bin $(10 \mathrm{~ms})$ comparisons of the raw STJH with two control matrices (see Fig. 2) (see Results, A method to examine correlated rhinal activity time locked to mPFC firing). The first control matrix was used to test that peaks in the STJH are indeed locked to $\mathrm{mPFC}$ firing. We generated surrogate $\mathrm{mPFC}$ spike trains with a similar number of spikes as in the original spike trains, but with spike times selected from a random homogenous distribution. The STJH was then recalculated and this process was repeated 50 times. The random STJHs were averaged to form the control matrix. The second control matrix tests that peaks in the STJH are not merely caused by independent rhinal responses to the mPFC (a "shift predictor"). To address this, the set of segments of perirhinal activity surrounding mPFC spikes was shuffled and then matched to the set of segments of entorhinal activity surrounding mPFC spikes. In other words, each segment of perirhinal activity around an mPFC spike was now matched to an entorhinal segment surrounding a different $\mathrm{mPFC}$ spike. The process was repeated 50 times, each time calculating a new $\mathrm{STJH}$, and the result averaged to form the control matrix. STJHs were considered significant if they differed from both control STJHs $[p<0.05$, Bonferroni corrected for the number of bins (900)]. When computing ratios of significant STJHs as a function of a behavioral event (CS or reward) during a trial, we used nonoverlapping windows of $0.5 \mathrm{~s}$. We also tested windows of $250 \mathrm{~ms}$ or $1 \mathrm{~s}$ and this produced qualitatively identical results.

Although visual inspection of the STJHs indicated that the vast majority of them had a single dominant peak, this was quantified by calculating the DIP statistical test for unimodality (Hartigan and Hartigan, 1985). Using this approach, we found that as many as $76 \%$ of the STJHs were unimodal.

Computation of directionality index. We computed a directionality index to assess the direction of impulse traffic in the rhinal cortices in relation to $\mathrm{mPFC}$ activity. This was obtained by separately adding all bins above the main diagonal $(a)$ versus below the main diagonal $(b)$ in the STJHs and then computing $(a-b / a+b)$ for each STJH. The directionality index ranges from -1 to 1 and a positive value indicates that the PR cell tended to fire before the ER cell and inversely for negative values.

Statistical significance of cross-correlograms. To determine whether cross-correlograms (CCs) were statistically significant, long epochs of spontaneous activity were segmented randomly. Then, we shuffled the segments of one neuron with respect to the other, and recomputed the CCs 100 times, thus generating 100 control CCs. Each center bin ( $\pm 50 \mathrm{~ms}$ ) from the original CC was compared against the randomized distribution for that bin. If any bin was significantly different at the $p<$ 0.05 level, the CC was deemed significant. We did not compensate for multiple comparisons of the number of center bins, thus biasing the results toward a higher proportion of significant CCs. Despite this, a low proportion of significant PR-ER CCs was observed (see Results).

Identifying aspects of $m P F C$ activity that correlate with significant STJHs. We considered three factors: (1) firing rate, (2) firing pattern (spike clustering), and (3) synchrony between simultaneously recorded mPFC cells. Spike clustering (2) was defined as the number of spikes generated 
with interspike intervals $\leq 50 \mathrm{~ms}$ as measured from autocorrelation functions. This is equivalent to computing interspike interval histograms. mPFC synchrony (3) was defined as the number of spikes in the $\pm 50 \mathrm{~ms}$ range of crosscorrelation functions. Auto- and crosscorrelograms were first normalized for variations in firing rates. To this end, spikes were shuffled 50 times and the auto- or crosscorrelation recalculated each time. These randomized correlograms were then averaged and subtracted from the original correlograms. The excess in activity (nonshuffled minus shuffled average) in the $\pm 50 \mathrm{~ms}$ center bins was then used below.

Next, we constructed a multiple logistic regression model using these three factors as independent variables and the significance of the STJHs as the dependent variable. It is important that such analyses not only include short post-CS or postreward periods, because the activity of mPFC cells might show large variations that are associated with the CS and/or rewards and therefore dominate the correlations in the model, although being unrelated to changes in the proportion of STJHs. Thus, to limit the impact of such coincidental relations, we calculated all of the required parameters during long periods of activity that surrounded and included the trace-conditioning trials: from $10 \mathrm{~s}$ before the CS to $15 \mathrm{~s}$ after the CS, analyzed in nonoverlapping $250 \mathrm{~ms}$ time windows. It should be pointed out that the results of the multiple regression held when the post-CS and postreward data were omitted from the analysis or when the data obtained from the early versus late stages of learning were considered separately.

For the population model, we collapsed data across sessions and cats, and calculated the proportion of significant STJHs in each time window, together with the average of each of the three independent parameters. After verifying that the complete model was significant (with a likelihood ratio test, $p<$ 0.05 ), we used a backward stepwise procedure that drops one variable at a time and checks if the difference between the complete model and the reduced one is significant (likelihood ratio test).

To take into account the variability between different cats, sessions, and cells or cell couples, we next calculated individual models that related the activity of individual mPFC cells or cell couples to the STJH(s) they contributed to. In this case, the dependent variable was binary (significant STJH or not), and the independent variables (1-3) were continuous. Logistic regressions were fitted using the Matlab glmfit function (Mathworks, Natick, MA).

\section{Results}

Using two arrays of high-impedance microelectrodes (Fig. $1 \mathrm{~A}$, dots), the activity of $\operatorname{mPFC}(n=125)$ (Fig. $1 B 1, C)$, ER $(n=145)$ (Fig. $1 B 2, C)$, and PR $(n=224)$ (Fig. $1 B 2, C)$ neurons was simultaneously recorded in behaving cats.

\section{A method to examine correlated rhinal activity time locked to mPFC firing}

Typically, coactivity patterns in two brain areas, in this case the PR and ER cortices, are assessed by computing CCs. With this method, the relation with a third factor, in this case the activity of mPFC neurons, is hidden in the result of the CCs and cannot be assessed. To circumvent this problem, we used an analysis tech-
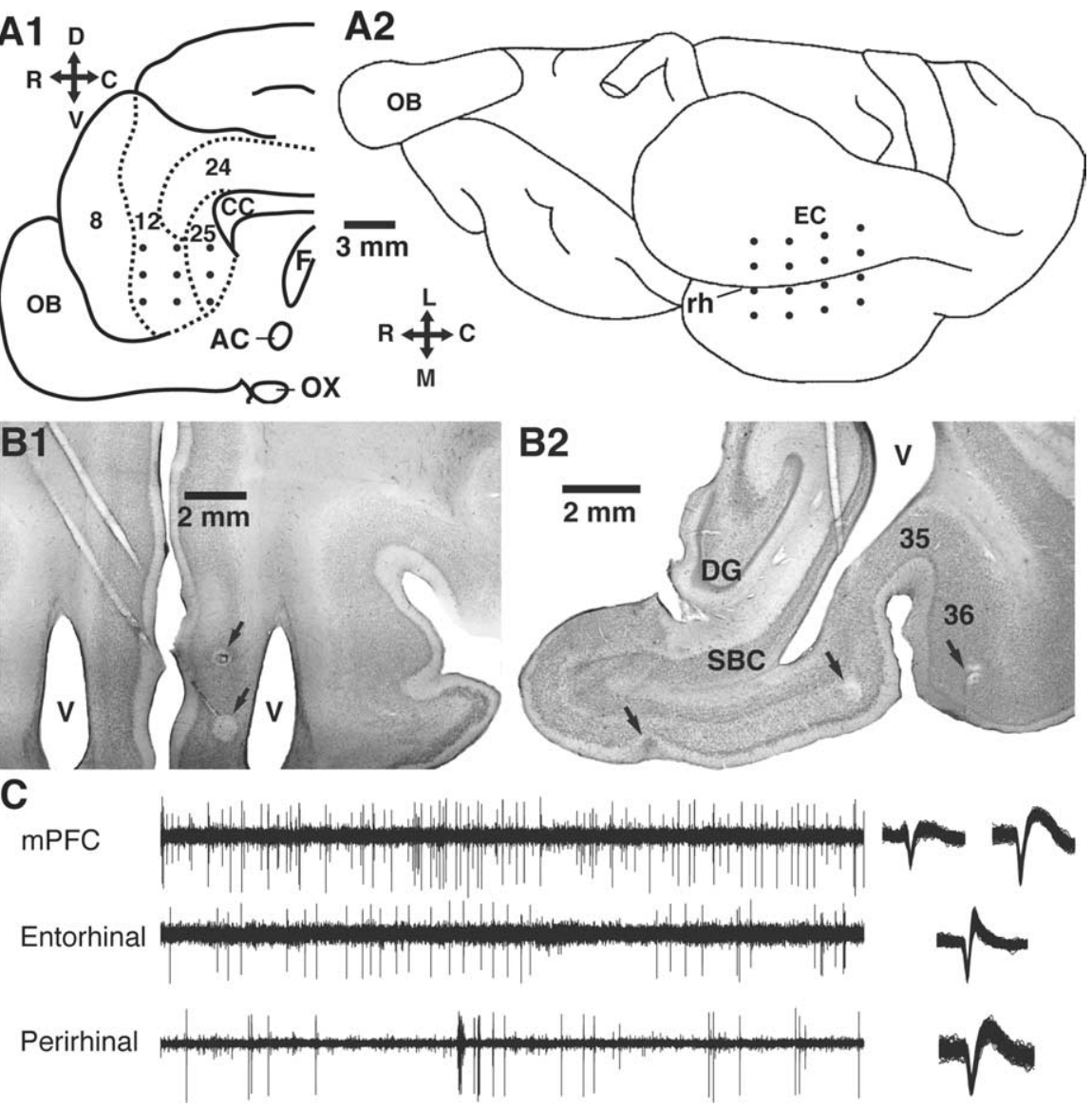

Figure 1. Simultaneous extracellular recording of $\mathrm{mPFC}, \mathrm{PR}$, and ER neurons. $\boldsymbol{A}$, Recording method. Arrays of high-impedance microelectrodes (dots) were inserted in the mPFC $(\boldsymbol{A} 1)$ and rhinal cortices $(\boldsymbol{A 2})$. $\boldsymbol{B}$, Histological verification of recording sites. Coronal brain sections showing the location of electrolytic lesions (arrows) performed at the end of the experiments to mark the

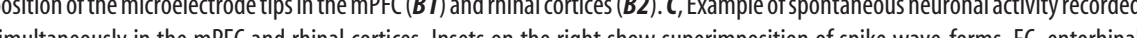
cortex; OB, olfactory bulb; rh, rhinal sulcus; L, lateral; $R$, rostral; M, medial; $C$, caudal; D, dorsal; V, ventral; DG, dentate gyrus; $S B C$, subiculum; F, fornix; $A C$, anterior commissure; OX, optic chiasma.

nique that measures correlated activity in the rhinal cortices, but in relation to $\mathrm{mPFC}$ firing. Specifically, we adapted the JPSTH method (Aertsen et al., 1989); but instead of using an external stimulus as the triggering event as in the JPSTH, we used the spikes generated by mPFC neurons. Hereafter, this analysis will be termed spike-triggered joint histogram (STJH) (Paz et al., 2006). We first explain how this analysis is performed and interpreted and then describe the results it yielded.

STJHs are computed by taking segments $( \pm 150 \mathrm{~ms})$ of rhinal activity around mPFC spikes. As shown in Figure $2 A$, the abscissa and ordinate of the STJHs respectively represent when PR and ER spikes occurred (red ticks) in relation to $\mathrm{mPFC}$ spikes (at time 0 on both axes). Then, STJH bins that contain a coincidence of spikes (Fig. 2A, squares) are incremented. In other words, if around a given mPFC spike, there was a spike from the PR cell at time $x$, and one from the ER cell at time $y$, one count is added to the matrix bin $(x, y)$. Repeating this process for all mPFC spikes gradually produces the raw STJH. One example of such an STJH is presented in Figure 2 B1, in which the counts are color coded. The STJHs can reveal correlations that are often invisible in a raw CC because standard CCs include all of the spikes that rhinal cells generate, whereas STJHs only consider those that occur in relation to $\mathrm{mPFC}$ action potentials.

Because we are interested in mPFC-related rhinal correla- 

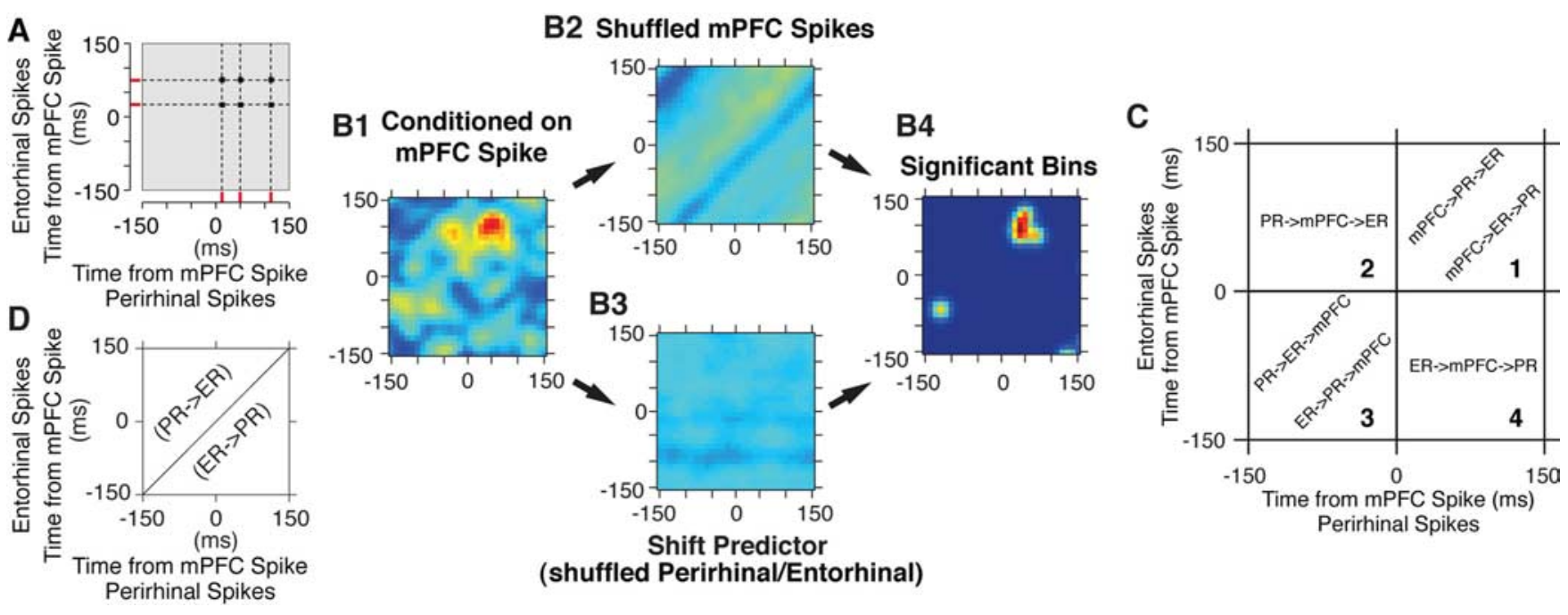

Figure 2. Methods used to construct and analyze STJHs. $\boldsymbol{A}$, Segments of ER ( $y$-axis) and PR ( $x$-axis) activity centered on mPFC spikes were isolated to identify bins that contained a coincidence (squares) of spikes (red ticks). Matrix bins containing coincident rhinal spikes (squares) were incremented and this process was repeated for all $\mathrm{mPFC}$ spikes, producing the STJH. $\boldsymbol{B}$, The actual STJH (B1) was compared with two sets of 50 control matrices $(\boldsymbol{B 2}, \boldsymbol{B} 3)$, one obtained by shuffling the $\mathrm{mPFC}$ spike trains $(\boldsymbol{B} 2)$ and the other by shuffling the mPFC spike train of one of the two rhinal cells (B3). Bins were considered significant when they differed from both randomly generated sets of values (B4). $\boldsymbol{C}$, The STJHs can be divided in four quadrants, each corresponding to a different firing sequence. $\boldsymbol{D}$, The location of significant bins with respect to the main diagonal indicates the prevalent direction of impulse traffic in the rhinal cortices.

tions, we tested the raw STJHs against two null hypotheses: (1) that the observed correlation is similar to that expected independently of mPFC activity; and (2) that the correlation reflects independent responses of rhinal neurons to mPFC activity. Two control randomized matrices were calculated for the two hypotheses (see Materials and Methods) (Fig. 2 B2,B3) and we performed bin-to-bin comparisons of significance between the raw and the two randomized sets of STJHs, using a Poisson distribution with a threshold $p$ value corrected for multiple comparisons (Fig. 2 B4). Finally, we tested whether most significant bins clustered around one peak (76\% of STJHs, DIP statistical test). All analyses were limited to these unimodal STJHs.

The location of significant bin clusters in the STJHs contains information about the timing of correlated rhinal activity in relation to mPFC spikes (Fig. 2C). A concentration of significant bins in quadrant 1 (Fig. 2C) represents correlated rhinal activity that occurred after $\mathrm{mPFC}$ spikes whereas a concentration of bins in quadrant 3 (Fig. 2C) implies that mPFC firing typically occurred after correlated rhinal spikes. For other possible timing sequences, see Figure 2C. To capture the particular coding scheme revealed by the STJHs, we summed the bins in each quadrant separately, and designated the largest as the timing index. Below, when comparing $\mathrm{mPFC}$-related rhinal interactions in different conditions, we compared the proportion of cell triplets with STJHs falling in the four quadrants. Because the sum of the proportions in the four quadrants is $100 \%$, a decrease in one quadrant is necessarily accompanied by an overall increase in the others.

The location of significant bins in the STJHs also indicates the prevalent direction of impulse traffic in the rhinal cortices in relation to $\mathrm{mPFC}$ spikes. Bins above the main diagonal of the STJH represent correlated activity where ER firing follows PR spikes or, in other words, lateral-to-medial impulse transfer (Fig. $2 D)$. Conversely, bins below the main diagonal indicate ER to PR transfer (Fig. 2D). Because the directionality of information transfer in the rhinal cortices has important implications for the mechanisms of learning and memory, we computed a directionality index (DI) that captures this dimension. To this end, we separately added all bins above the main diagonal ( $a$ ) versus below the main diagonal $(b)$ in the STJHs (Fig. $2 D$ ). The DI was obtained by computing $(a-b / a+b)$ and thus ranges from -1 to 1 , where a positive value indicates that the PR neuron tended to fire before the ER cell and inversely for negative values.

\section{mPFC-related modulation of rhinal interactions}

We first consider mPFC-related rhinal interactions taking place during the quiet waking state, when no rewards were delivered. Previous studies that cross-correlated the activity of PR and ER neurons during the waking state reported a low incidence of significant CCs (Pelletier et al., 2004). This was also the case here, where only $14 \%$ (23 of 164) of PR-ER cell couples had significant CCs $(p<0.05$, shuffling procedure). However, when we used STJHs to restrict the analysis to rhinal spikes that occurred around $\mathrm{mPFC}$ activity, we observed significant rhinal correlations that were not apparent in the CCs. This point is illustrated in Figure 3, which shows cross-correlograms for four couples of PR and ER neurons (Fig. 3A), the corresponding STJHs centered on mPFC spikes (Fig. $3 B$ ), and control STJHs produced by shuffling of the mPFC spike trains (Fig. $3 C$ ). As shown in Figure $3 A$, a majority of cross-correlograms were flat (Fig. 3A1-A3), indicating that there is little correlated activity between rhinal neurons when mPFC activity is not taken into account. In contrast, all depicted STJHs (Fig. 3B) show that in relation to MPFC spikes, there is a high degree of correlated PR-ER activity. Moreover, even a high raw rhinal cross-correlogram (Fig. 3A4) can actually be related to $\mathrm{mPFC}$ spikes (Fig. 3B4), because the increased correlation is evident immediately after $\mathrm{MPFC}$ firing in the STJH.

Using the criteria described above on all cell triplets $(n=164)$, $35 \%$ (or 58 of 164) of STJHs were deemed statistically significant, much more than expected by chance $(p<0.0001$, Fisher's exact test). When this ratio was computed separately for couples of rhinal cells where CCs were significant or not, a similar percentage of significant STJHs was observed (42 and 34\%, respectively). Thus, the STJH analysis indicates that hidden in the CCs, are periods of enhanced rhinal interactions that prevalently occur when $\mathrm{mPFC}$ cells are active. To gain some insight in the propor- 
tion of rhinal spikes involved in this effect, we compared the number of PR and ER spikes occurring within $\pm 150 \mathrm{~ms}$ of $\mathrm{mPFC}$ spikes versus random times. We found that PR and ER cells were slightly more active around $\mathrm{mPFC}$ spikes compared with random times (an increase of 2.6 and $4.3 \%$, respectively). Expressed in firing rates, $\mathrm{PR}$ and ER cells fired at 1.32 and 2.36 $\mathrm{Hz}$, respectively, around random times compared with 1.35 and $2.46 \mathrm{~Hz}$ around $\mathrm{mPFC}$ spikes. These small elevations suggest that the critical factor behind the effect disclosed by the STJHs is a higher incidence of temporally contiguous PR and ER spikes, not a global increase in rhinal activity levels.

Figure $4 A$ illustrates the grand average of significant STJHs during the waking state. It shows that correlated PR-ER firing prevalently occurred after $\mathrm{mPFC}$ spikes. To examine this further, we studied the distribution of the timing index (Fig. 2C) where significant bins in each quadrant of the STJHs are summed separately, and the quadrant with the highest total is designated as the timing index. This revealed that peaks of correlated rhinal activity were not distributed homogeneously in relation to the mPFC spikes (Fig. $4 B)\left(p<0.01, \chi^{2}\right)$. Rather, many STJHs had a preponderance of significant bins in the first quadrant $33 \%$ higher than expected; $p<0.05, \chi^{2}$ ), where mPFC spikes generally occurred before PR and ER firing. In contrast, $22 \%$ of significant STJHs fell in the third quadrant, where mPFC spikes usually occurred after correlated rhinal activity.

To determine whether there was a preferential directionality of rhinal interactions in relation to $\mathrm{mPFC}$ activity, we computed the DI for all significant STJHs (Fig. 4C). Both positive and negative DIs were extensively represented in the population, with an average slightly, but significantly below zero (population mean, -0.01 ; $t$ test, $p<0.05)$. Thus, in quiet waking, both directions of PR-ER interactions occur in relation to MPFC activity, but with a slight preference for ER to PR communication.

\section{Learning a trace-conditioning task}

To study how mPFC-rhinal interactions are affected during memory formation, three cats were trained on an appetitive trace-conditioning task (Fig. 5A). In this task, a visual CS that predicted the delivery of a liquid reward $3 \mathrm{~s}$ later was presented at random intervals (30-90 s) (Fig. 5A, methods). The CS ( $1.5 \mathrm{~s}$ ) was a global change in the illumination of a computer screen that encompassed most of the visual field of the animals. The reward was a highly salient event for the animals
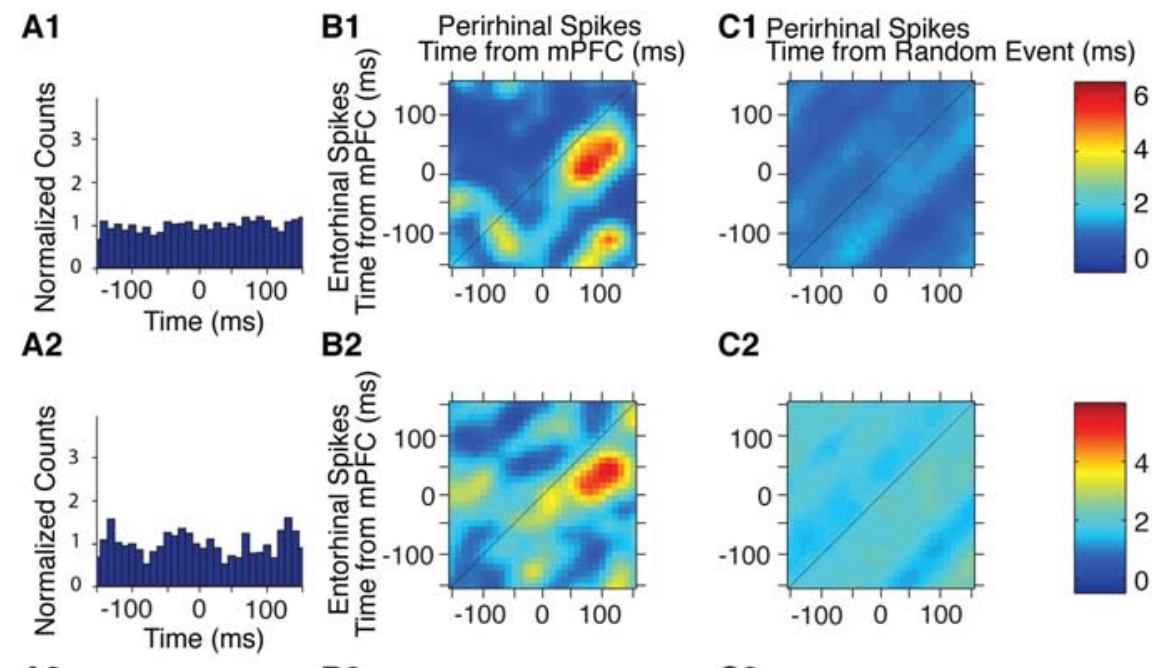

A3

B3

C3
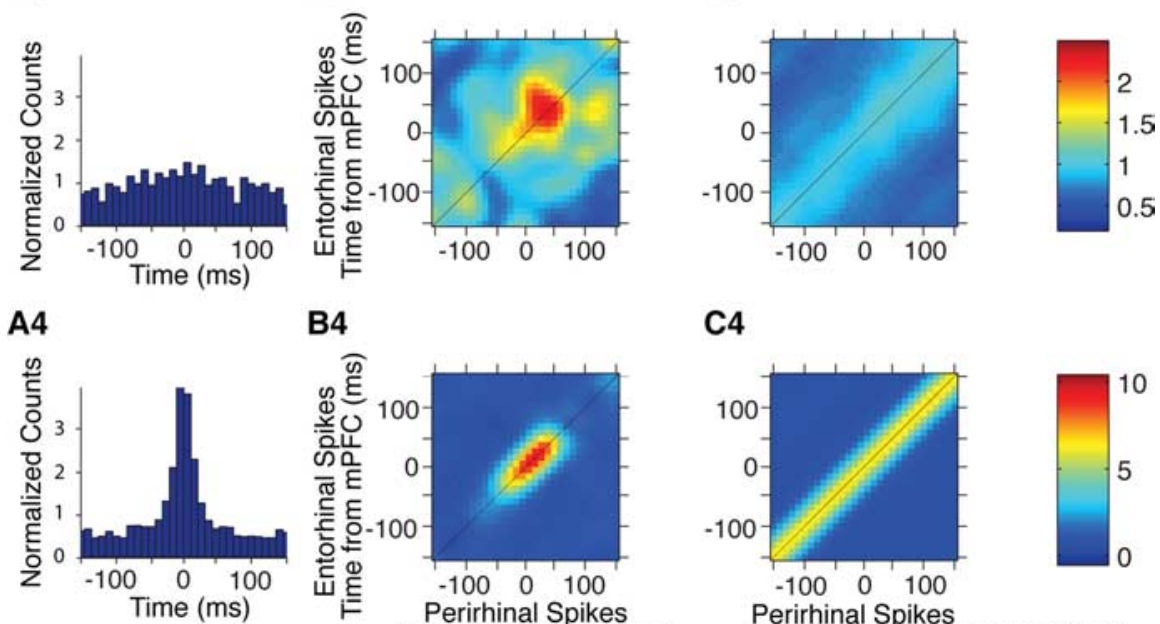

B4

C4
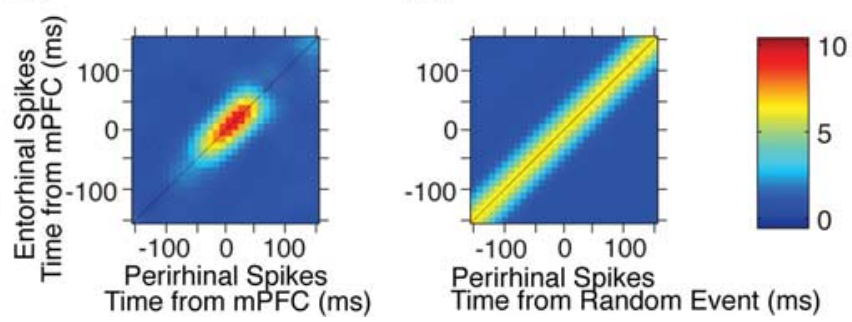

Figure 3. $\mathrm{mPFC}$-related enhancement of correlated rhinal firing during quiet waking. A1-C4, Four couples of simultaneously recorded PR and ER neurons. $A$, Cross-correlation of spontaneous activity. $B$, STJHs for the same cell couples (ER, $y$-axis; PR, $x$-axis), but conditioned on the activity (time 0 ) of a simultaneously recorded $\mathrm{mPFC}$ cell. $\boldsymbol{C}$, Control STJHs computed after shuffling the $\mathrm{mPFC}$ spike train 50 times and averaging the result.
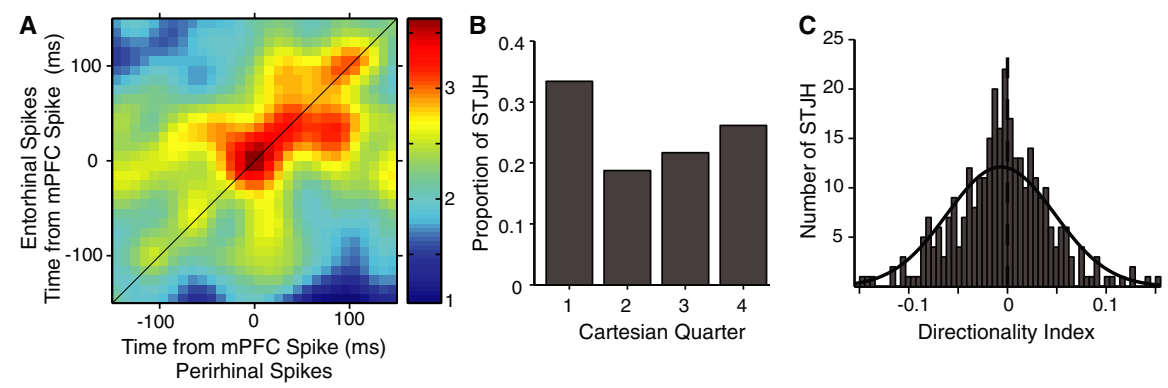

Figure 4. Spontaneous neuronal interactions revealed by the STJHs during quiet waking. $\boldsymbol{A}$, Grand average of all STJHs. $\boldsymbol{B}$, Distribution of the timing index. Each significant STJH was ascribed a number from 1 to 4 depending on the quadrant where significant bins were concentrated (Fig. 2C). C, Distribution of the directionality index in all significant STJHs. The directionality index was obtained by separately adding all bins above $(a)$ versus below $(b)$ the main diagonal in the STJHs and then computing $(a-b / a+b)$ for each STJH. A positive value indicates that the PR neuron tended to fire before the ER cell and inversely.

because it was preferred food and the subjects were fed only during the recording sessions. The animals underwent one training session per day (38-95 trials/session) over a period of $9 \mathrm{~d}$.

In addition to the data reviewed in the Introduction, much evidence indicates that this task is dependent on the hippocam- 

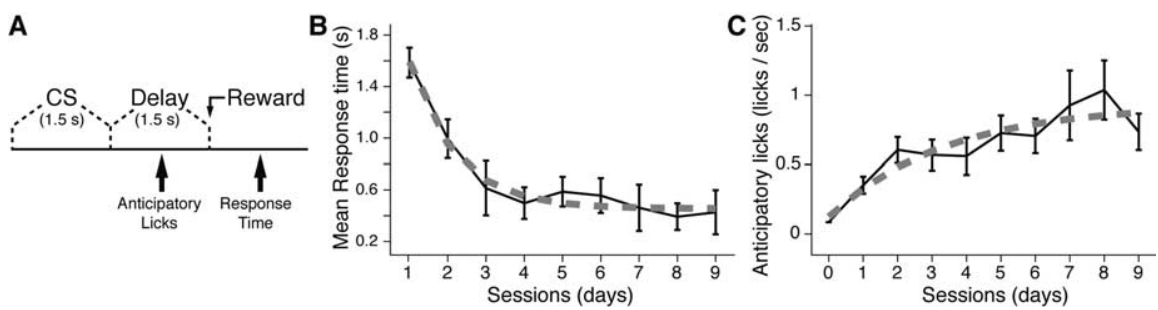

Figure 5. Learning progression in an appetitive trace-conditioning paradigm. $\boldsymbol{A}$, Scheme showing the relative timing of the visual $C S$, delay period, and reward delivery in the trace-conditioning paradigm used in the present study. Two behavioral measures were used to assess learning: the rate of anticipatory licking during the delay period and the mean latency to completion of the first 25 licks after reward delivery (termed response time here). $\boldsymbol{B}$, Graph plotting the mean response time ( $y$-axis) as a function of training sessions ( $x$-axis; one per day). $C$, Graph plotting the rate of anticipatory licking ( $y$-axis) as a function of training sessions ( $x$-axis). Error bars indicate SEM.

pus and mPFC. First, in eye blink conditioning, introduction of a delay between the conditioned and unconditioned stimuli renders this task hippocampal-dependent (Shors, 2004). Second, in several appetitive operant learning tasks involving a delay between the conditioned stimuli and responses, hippocampal lesions interfere with learning (Savage et al., 2004). Third, in an appetitive trace-conditioning paradigm very similar to the one used here, disruption of hippocampal activity with intraseptal infusions of scopolamine interfered with learning (Asaka et al., 2000). Last, hippocampal and mPFC neurons exhibit learningdependent changes in activity profile in relation to the conditioned stimulus and during the delay period (Berger et al., 1983; Solomon et al., 1986; Weiss et al., 1996; Munera et al., 2001; Baeg et al., 2001; McEchron et al., 2003; Gilmartin and McEchron, 2005).

Two behavioral measures were used to assess learning on our task: the mean response time, defined as the mean latency of the first 25 licks after reward delivery (Fig. 5B), and the rate of anticipatory licking after CS onset but before reward delivery (Fig. $5 C$ ). Both measures showed the same trend for all cats considered individually or as a group: a rapid initial learning phase that could be observed as early as training days $2-3$, followed by a protracted period of slower improvements that extended up to training days 5-9 (ANOVA of trials over sessions, $p<0.05$ for all cats and both measures). In keeping with this, the two learning curves were well fitted with an exponential function (Fig. $5 B-C$, dashed lines) ( $p<0.01$ for both, bootstrap)

Overall, these behavioral measures suggest that although memory for the predictive value of the CS is formed as early as the second training session, it is then gradually strengthened over a period of several days.

\section{Learning-related changes of mPFC-rhinal interactions}

Although our sample of mPFC neurons included infralimbic (IL; $n=76)$ and prelimbic (PL; $n=49)$ cells, the results obtained with these two groups of cells were pooled because (1) IL and PL have overlapping projections to the rhinal cortices (Vertes, 2004), and (2) the activity profile of IL and PL neurons during the CS, delay, and reward were statistically indistinguishable (supplemental Table 1, available at www.jneurosci.org as supplemental material).

To determine whether $\mathrm{mPFC}$-rhinal interactions fluctuated as a function of learning, we computed STJHs during the traceconditioning trials, in nonoverlapping $0.5 \mathrm{~s}$ windows for all triplets of simultaneously recorded mPFC, PR, and ER neurons $(n=$ 164). Immediately after CS presentation, the ratio of significant STJHs increased markedly (Fig. 6A) (from 21 to $41 \%$; $p<0.001$, $\left.\chi^{2}\right)$, indicating an increase in the strength of mPFC-related rhinal correlations. Reward delivery was also associated with an increase in the proportion of significant STJHs (Fig. 6B) (from 19 to $\left.42 \% ; p<0.001, \chi^{2}\right)$. Additionally, the distribution of the timing index was altered, with a decrease in the third quadrant (from 30 to $13 \%$ after CS and from 25 to $11 \%$ after reward; $p<0.05$ for both, $\chi^{2}$ ), suggesting that rhinal correlations follow $\mathrm{mPFC}$ activity in a higher proportion of STJHs.

To study the time course of these changes and their relation to behavior, we examined how the proportion of significant STJHs fluctuated across training sessions, pooling the data obtained during the first $1.5 \mathrm{~s}$ after CS onset. This revealed a gradual increase in the proportion of significant STJHs as a function of training sessions (Fig. 6C) (exponential fit, $p<0.01 ; \chi^{2}, p<0.01$ ). This was in contrast to reward-related activity where no temporal trend was observed (Fig. 6D) ( $p>0.1)$.

To further establish that the findings were specific to perirhinal-entorhinal interactions, we computed STJHs on rhinal neurons located in the same area, but located at different rostrocaudal levels. Thus, in this case, the STJHs were still conditioned on mPFC spikes, but instead of analyzing the activity of perirhinal and entorhinal neurons, we examined the activity of two perirhinal or two entorhinal cells. We found that although the baseline proportions of significant STJHs were similar (19\% for the CS, $20 \%$ for the reward), the CS- and reward-related increases in the proportion of significant STJHs were much lower (increase of 6 and $7 \%$ for the CS and reward, respectively) compared with $\sim 20 \%$ for triplets including mPFC, perirhinal, and entorhinal cells ( $p<0.001$, Fisher's exact test).

Overall, these results indicate that reward delivery causes a consistent increase in mPFC-related rhinal correlations at all phases of learning, whereas CS-related increases in rhinal correlations develop gradually, as learning progresses and the CS acquires predictive value. In support of this, the exponential fits for the progression of behavior (Fig. $5 B, C$ ) and the ratio of significant STJHs during the CS (Fig. 6C) had similar time constants $(p>0.1, t$ test).

\section{Learning-related changes in the directionality of mPFC- related rhinal interactions}

Next, to test whether learning-related changes in the proportion of significant STJHs were associated with a shift in the directionality of rhinal interactions, we examined how the DI fluctuated in relation to the CS and reward in $0.5 \mathrm{~s}$ windows around the events of interest (Fig. $6 E-H$ ). To do so, we quantified the proportion of STJHs with positive DIs. A proportion $>50 \%$ indicates that the prevalent direction of impulse traffic in the rhinal cortices is from PR to ER neurons, whereas a proportion $<50 \%$ signifies the opposite. After the CS, a significant reduction from the $50 \%$ baseline was observed (Fig. $6 E)\left(p<0.01, \chi^{2}\right)$, indicating a preferential ER to PR direction of impulse transfer around $\mathrm{MPFC}$ activity. In contrast, no change in DI was seen in relation to the reward (Fig. $6 F)\left(p>0.1, \chi^{2}\right)$.

To study the time course of DI fluctuations as a function of the learning phase, we pooled the data obtained during the first $1.5 \mathrm{~s}$ after CS onset (Fig. 6G) or reward delivery (Fig. 6H). This analysis revealed a similar trend as for the $\mathrm{STJH}$ ratio analysis (Fig. $6 C)$ : as learning progressed, there was a gradual shift from no 

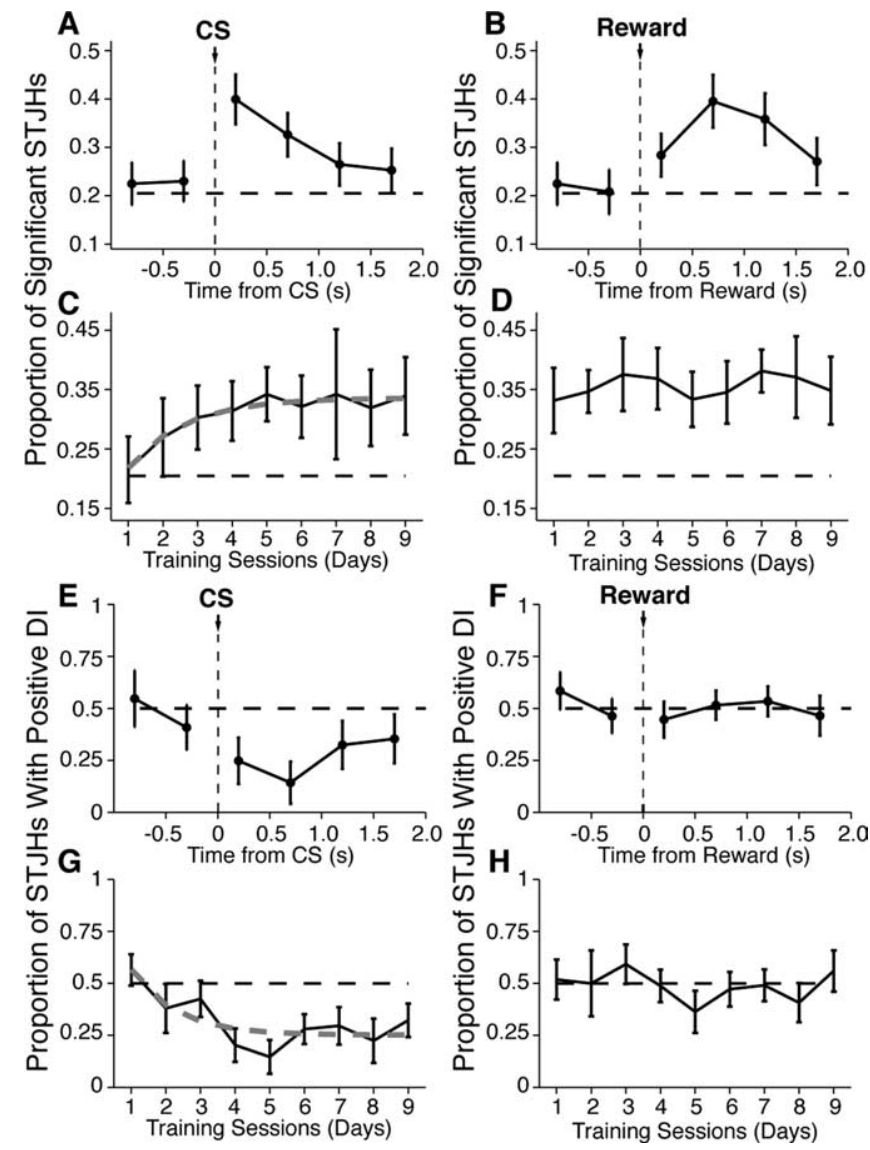

Figure 6. $\boldsymbol{A}-\boldsymbol{H}$, Learning-related variations in the proportion of significant STJHs $(\boldsymbol{A}-\boldsymbol{D})$ and DI $(\boldsymbol{E}-\boldsymbol{H}) . \boldsymbol{A}, \boldsymbol{B}$, Proportion of significant STJHs ( $y$-axis) averaged over all training sessions as a function of time ( $x$-axis) around $C S$ onset $(\boldsymbol{A})$ and reward delivery $(\boldsymbol{B}) . \boldsymbol{C}, \boldsymbol{D}$, Fluctuations in the proportion of significant STJHs ( $y$-axis) as a function of daily training sessions ( $x$-axis in days). $\boldsymbol{E}$, $\boldsymbol{F}$, Proportion of significant STJHs with positive DIs ( $y$-axis) as a function of time ( $x$-axis) around CS onset $(\boldsymbol{E})$ or reward delivery $(\boldsymbol{F}), \boldsymbol{G}, \boldsymbol{H}$, Fluctuations in the proportion of significant STJHs with positive DIs ( $y$-axis) as a function of daily training sessions ( $x$-axis in days). The data in $\mathbf{C}, \boldsymbol{D}, \mathbf{G}$, and $\boldsymbol{H}$ were obtained by pooling the activity recorded in $1.5 \mathrm{~s}$ windows after $C S$ onset $(\boldsymbol{C}, \boldsymbol{G})$ or reward delivery $(\boldsymbol{D}, \boldsymbol{H})$. All panels show average \pm SEM of data obtained in three cats.

directional propensity to a preference for mediolateral interactions (Fig. 6G) (exponential fit, $p<0.01 ; \chi^{2}, p<0.01$ ). This was in contrast to reward related activity where the DI did not fluctuate with the learning phase (Fig. $6 H)(p>0.01)$.

To summarize, whereas there was no preferential directionality of PR-ER interactions in relation to rewards at all phases of learning, in relation to the CS, ER to PR impulse transfer gradually became prevalent as learning progressed.

\section{What aspects of mPFC activity promote rhinal correlations?}

To address this question, we asked what parameters of $\mathrm{mPFC}$ activity correlate with the emergence of significant STJHs. First, higher mPFC firing rates could facilitate rhinal correlations (although STJH were normalized for firing rates). Second, increased clustering of the spikes generated by individual mPFC cells could participate by allowing for enhanced temporal summation of inputs into the rhinal cortices. To assess this possibility, we considered the proportion of interspike intervals $\leq 50$ ms generated by mPFC cells $(n=124)$. Finally, if the discharges of different mPFC neurons were more synchronized, this could facilitate rhinal interactions via summation of inputs. Thus, to take this factor into account, we cross-correlated the activity of simultaneously recorded mPFC neurons ( 181 cell couples) and summed the activ- ity in the center bins $( \pm 50 \mathrm{~ms}$ ). CCs were normalized for firing rate variations using a shuffling procedure (see Materials and Methods).

To investigate the contribution of these three different parameters to the occurrence of $\mathrm{mPFC}$-facilitated rhinal transmission, we constructed a regression model that included synchrony, spike clustering, and firing rate as independent parameters (Fig. $7 A-C, x$-axes, respectively), and the ratio of significant STJHs as the dependent variable (Fig. $7 A-C, y$-axis). These parameters were calculated in nonoverlapping $0.25 \mathrm{~s}$ windows (from $10 \mathrm{~s}$ before to $15 \mathrm{~s}$ after the CS; similar results were obtained when these analyses were performed only on pre-CS, post-CS, or postreward periods).

At the population level, the multiple regression model (including all three independent parameters) was significant $(p<$ 0.01 , likelihood ratio test). A backward stepwise procedure that dropped one variable at a time revealed that both increased synchrony among simultaneously recorded mPFC cells $(p<0.01$, likelihood ratio test) (Fig. $7 A$ ), as well as higher clustering of the spikes generated by individual mPFC neurons $(p<0.05)$ (Fig. $7 B)$, contributed significantly. In contrast, this procedure revealed that the firing rate of $\mathrm{mPFC}$ neurons was not a significant contributor $(p=0.2)$ (Fig. $7 C$ ).

Last, to consider the variability between cells or cell couples, we performed separate regressions for each individual STJH $(n=$ 124). A representative example is shown in Figure $7 D-F$. The distribution of $p$ values for the three factors for these individual models revealed that firing rate, $\mathrm{mPFC}$ spike clustering, and mPFC synchrony had significant effects $(p<0.05)$ in 12,18 , and $27 \%$ of cases, respectively. The difference between these percentages was significant $\left(p<0.01, \chi^{2}\right)$.

Overall, these analyses suggest that several interacting aspects of mPFC activity contribute to enhance rhinal correlation, particularly increases in the synchrony and, to a lesser extent, in the clustering of $\mathrm{mPFC}$ action potentials.

\section{Discussion}

The present study was undertaken to examine the relative timing of $\mathrm{mPFC}$ and rhinal activity during the acquisition of a hippocampal-dependent trace-conditioning task. The importance of this issue stems from findings implicating the mPFC in the consolidation of hippocampal-dependent memories and evidence suggesting that an mPFC-driven modulation of neocortical-hippocampal interactions at the level of the rhinal cortices might underlie this effect. Our results indicate that in relation to $\mathrm{mPFC}$ activity, rhinal interactions are facilitated. At early stages of learning, this effect was most pronounced in relation to reward whereas at late learning stages, it also occurred in relation to the CS. Moreover at late, but not early stages of learning, CS-related mPFC activity was prevalently associated with enhanced transmission from ER to PR neurons. Because the ER to PR connection is an obligatory step in the multisynaptic pathway that links the hippocampus to the neocortex, these results suggest that mPFC activity facilitates rhinal transfer of hippocampal impulses toward the neocortex. The significance of these findings for the consolidation of hippocampal-dependent memories is considered below.

\section{Learning-related changes in mPFC-rhinal interactions}

The observation that hippocampal lesions produce a temporally graded amnesia for recently learned events (Zola-Morgan and Squire, 1986; Kim and Fanselow, 1992) has led to the view that the hippocampal network forms rapid representations of events 

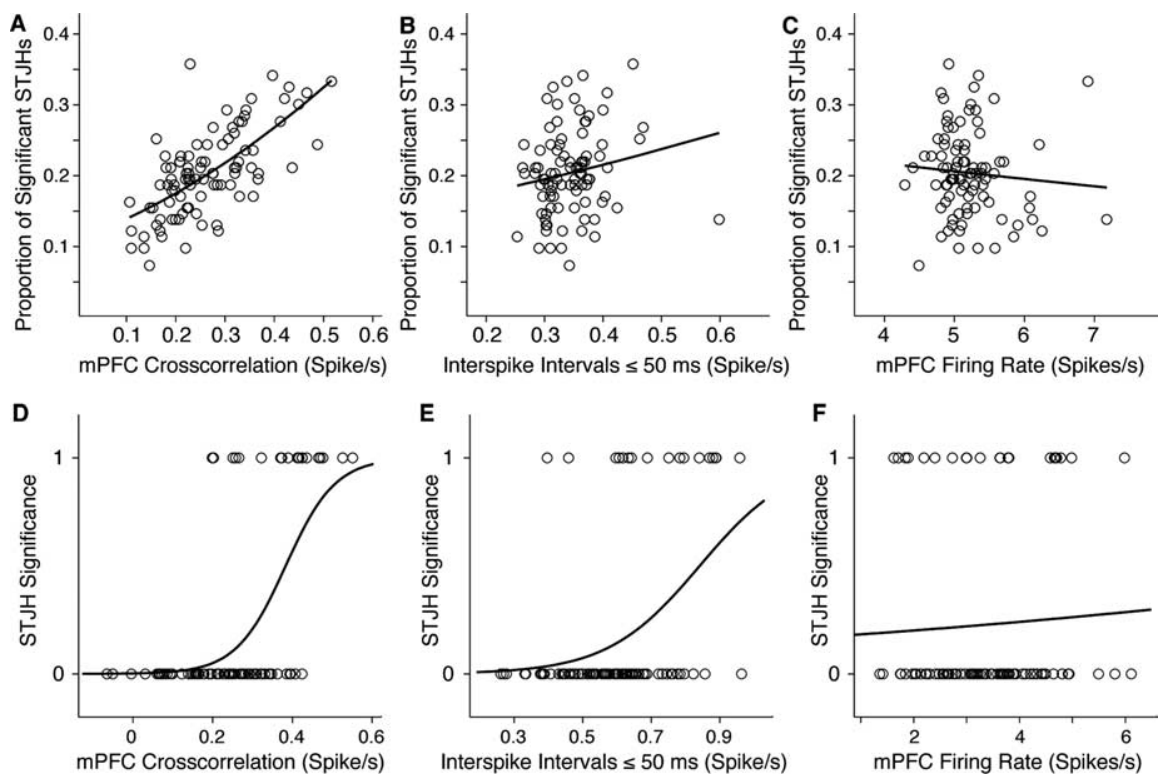

Figure 7. Aspects of mPFC activity that correlate with facilitated rhinal interactions. $\boldsymbol{A}-\boldsymbol{C}$, Graphs plotting the proportion of significantSTJHs in the $y$-axis, where each data point represents this proportion in $250 \mathrm{~ms}$ time windows spanning from 10 s before the CS to $15 \mathrm{~s}$ after the CS. $\boldsymbol{A}$, Variations in the proportion of significant STJHs ( $y$-axis) are related to synchrony within the mPFC $(x$-axis), as measured by cross-correlations between couples of simultaneously recorded mPFC cells ( $\pm 50 \mathrm{~ms}$ around the origin). The solid line is the logistic regression fit ( $p<0.001$, likelihood ratio test). $\boldsymbol{B}$, Variations in the proportion of significant STJHs ( $y$-axis) are related to changes in firing pattern ( $x$-axis, number of mPFC spikes generated with interspike intervals $\leq 50 \mathrm{~ms}$ derived from autocorrelations, $p<0.05$ ). $C$, The proportion of significant STJHs ( $y$-axis) is plotted against the instantaneous firing rate of $\mathrm{mPFC}$ cells $(x$-axis, $p>0.1)$. $\boldsymbol{A}-\boldsymbol{C}$, Each data point represents the average over cats, sessions, and cell couples $(\boldsymbol{A})$ or cells $(\boldsymbol{B}$, C. $\boldsymbol{D}-\boldsymbol{F}$, Example of one STJH. Each data point corresponds to a $250 \mathrm{~ms}$ window and the $x$-axes are the same as in $\boldsymbol{A}-\boldsymbol{C}$. The $\boldsymbol{y}$-axis denotes whether the STJH was significant (1) or not (0) in that window. Solid lines are the logistic regression fit $(p<0.001, p<$ 0.01 , and $p=0.8$ for $\boldsymbol{D}-\boldsymbol{F}$, respectively). The reason why the data are binary in $\boldsymbol{D}-\boldsymbol{F}$ and continuous in $\boldsymbol{A}-\boldsymbol{C}$ is that in the former case we related $\mathrm{mPFC}$ synchrony, firing pattern, and firing rate to the significance of a single STJH (a binary variable), whereas in the latter case, we related these parameters to the overall proportion of significant STJHs (a continuous variable).

apses onto principal rhinal neurons (Apergis-Schoute et al., 2006).

Our results support and extend these observations. Indeed, we found that at late, but not early stages of learning, CSevoked mPFC activity is associated with facilitated interactions between the ER and PR cortices. Importantly, this facilitation occurred in a specific direction: from ER to PR neurons, as would be expected for an mPFC-facilitated rhinal transfer of hippocampal information toward the neocortex. Note that this is consistent with the fact that mPFC afferents to the ER cortex focus on deep layers, where laterally directed projections originate (Burwell and Witter, 2002). The fact that there was no preferential directionality in relation to rewards suggests that the direction of facilitated rhinal interactions is not entirely determined by mPFC inputs, but also by the pattern of afferent activity that prevails in the rhinal cortices when mPFC neurons are engaged.

What mechanism could explain the gradual appearance of the CS-related mPFC facilitation of rhinal interactions? It is possible that during repeated training sessions, direct hippocampal projections to the mPFC gradually induce a potentiation of afferents conveying CS information to mPFC neurons (Sierra-Mercado et al., 2006). As a result, subsequent presentations of the CS would directly activate

to be remembered. Subsequently, through repeated interactions between the hippocampus and neocortex, the links between distributed neocortical representations would become strong enough to support long-term storage of the new information (Squire and Alvarez, 1995; Buzsaki, 1996; Sutherland and McNaughton, 2000). It was proposed that this gradual transfer of new information to the neocortex is required for their integration in existing memory networks as a more rapid transfer would corrupt the old memories (McClelland et al., 1995).

What are the anatomical substrates of hippocampalneocortical interactions? Most hippocampal projections to the neocortex involve a stepwise progression of impulses through the ER cortex and PR areas 35-36 before reaching the neocortex (Burwell and Witter, 2002). However, because impulse transfer in this multisynaptic pathway is subjected to strong inhibitory pressures (Biella et al., 2001; Martina et al., 2001; Pinto et al., 2006), rhinal transfer of hippocampal and neocortical information occurs with a low probability (Biella et al., 2002; Pelletier et al., 2004, 2005; de Curtis and Pare, 2004).

A notable exception to this indirect scheme of hippocamponeocortical connectivity is the $\mathrm{mPFC}$, which receive monosynaptic inputs from CA1 pyramidal cells (Swanson, 1981; Ferino et al., 1987; Jay and Witter, 1991; Thierry et al., 2000). Importantly, most hippocampal neurons that project to the MPFC have axon collaterals ending in the ER cortex (Swanson, 1981). In turn, the $\mathrm{mPFC}$ is in a key position to influence rhinal transfer of hippocampal inputs to the neocortex because it sends strong projections to the PR and ER cortices (Room et al., 1985; Sesack et al., 1989; Hurley et al., 1991), most of which involve excitatory syn-
mPFC neurons, which in turn would facilitate rhinal transfer of hippocampal inputs to the neocortex. This might occur both when the CS is presented and during slow-wave sleep, when hippocampal memories are thought to be "replayed" (Buzsaki, 1989; Sutherland and McNaughton, 2000).

Although the electrodes were lowered $100 \mu \mathrm{m}$ each day in the present study, it is unlikely that the activity modifications we observed were attributable to the fact that we sampled neurons from different layers. Indeed, depending on the cues, mPFCrelated rhinal activity varied or was stable across training sessions. For instance, whereas the CS-related increase in the proportion of significant STJHs and the associated shift in directionality developed gradually, no temporal trend was seen in relation to the reward for both parameters. Second, the results we obtained were very different from the ones seen when the STJHs were conditioned on basolateral amygdala (BLA) spikes (Paz et al., 2006). Finally, in the latter study, we recorded during a behavioral paradigm that consisted of unexpected rewards during the whole recording period. Although the electrodes were lowered just as in the current study, the increase in the proportion of significant STJHs seen in relation to these unexpected rewards did not change, indicating that (at least for BLA-based STJHs) the findings cannot be attributed to the fact that we recorded neurons in different layers.

\section{Comparison between the impact of mPFC and amygdala activity on rhinal interactions}

The relationship between the activity of $\mathrm{MPFC}$ and rhinal neurons evidenced here contrasts with that observed previously be- 
tween amygdala and rhinal cells (Paz et al., 2006). Indeed, it was found that BLA activity facilitates rhinal interactions in relation to reward delivery, but in a direction opposite to that seen with mPFC firing: from PR to ER cells. Also different from the present results was the fact that this effect was most pronounced in the early stages of learning, when the reward was still unexpected. At this late phase of learning, after the association between CS and rewards was acquired, the BLA-related facilitation of rhinal interactions occurred when the cats were anticipating the reward, but at a much longer post-CS latency than seen in relation to $\mathrm{mPFC}$ activity. Moreover, consistent with the idea that the BLA and $\mathrm{mPFC}$ play distinct roles in memory formation, the CS-related increase in the proportion of significant STJHs developed earlier for BLA than mPFC spikes. Indeed, the BLA effect reached a plateau at the third training session (Paz et al., 2006) compared with the sixth training session for the $\mathrm{MPFC}$ (present results).

The contrasting time dependence of $\mathrm{MPFC}$ and amygdalarelated effects in the rhinal cortices parallels the results of lesion and pharmacobehavioral studies (McGaugh, 2004). Indeed, both approaches suggest that amygdala activity mainly facilitates memory during and shortly after encoding whereas the mPFC contributes at longer delays. These observations are consistent with the opposite directionality of the MPFC and amygdala effects in the rhinal cortices. The amygdala-related facilitation of PR to ER communication (rhinal transfer of neocortical inputs to the hippocampus) is consistent with a facilitation of memory encoding whereas the MPFC-related facilitation of ER to PR interactions (rhinal transfer of hippocampal inputs to the neocortex) suggest that it enhances memory consolidation.

Finally, there is a confounding factor when interpreting our study: the behavioral paradigm we used does not dissociate between consolidation and task performance at late stages of acquisition. Because the mPFC and rhinal cortices are known to be involved in the latter process as well (see Introduction), our results could indicate that the mPFC, by facilitating transfer of information within the rhinal cortices, supports task performance (rather than memory consolidation) at these late stages of training. More work will be needed to address this distinction.

\section{Conclusions}

Combined with the lesion and functional imaging studies reviewed in the Introduction, our results support the idea that the $\mathrm{mPFC}$, by facilitating rhinal transfer of hippocampal inputs to the neocortex, is an important player in the slow iterative consolidation process supporting the integration of hippocampaldependent memories into neocortical networks. Coupled to the results obtained in the BLA (Paz et al., 2006), our observations reinforce the view that memory formation engages a distributed network of structures, each contributing in different ways to the formation and consolidation of memories.

\section{References}

Aertsen AM, Gerstein GL, Habib MK, Palm G (1989) Dynamics of neuronal firing correlation: modulation of "effective connectivity." J Neurophysiol 61:900-917.

Apergis-Schoute J, Pinto A, Pare D (2006) Ultrastructural organization of medial prefrontal inputs to the rhinal cortices. Eur J Neurosci 24:135-144.

Asaka Y, Seager MA, Griffin AL, Berry SD (2000) Medial septal microinfusion of scopolamine disrupts hippocampal activity and trace jaw movement conditioning. Behav Neurosci 114:1068-1077.

Baeg EH, Kim YB, Jang J, Kim HT, Mook-Jung I, Jung MW (2001) Fast spiking and regular spiking neural correlates of fear conditioning in the medial prefrontal cortex of the rat. Cereb Cortex 11:441-451.

Berger TW, Rinaldi PC, Weisz DJ, Thompson RF (1983) Single-unit analy- sis of different hippocampal cell types during classical conditioning of rabbit nictitating membrane response. J Neurophysiol 50:1197-1219.

Biella G, Uva L, de Curtis M (2001) Network activity evoked by neocortical stimulation in area 36 of the guinea pig perirhinal cortex. J Neurophysiol 86:164-172.

Biella G, Uva L, de Curtis M (2002) Propagation of neuronal activity along the neocortical-perirhinal-entorhinal pathway in the guinea pig. J Neurosci 22:9972-9979.

Bontempi B, Laurent-Demir C, Destrade C, Jaffard R (1999) Timedependent reorganization of brain circuitry underlying long-term memory storage. Nature 400:671-675.

Burwell RD, Witter MP (2002) Basic anatomy of the parahippocampal region in monkeys and rats. In: The parahippocampal region. (Witter MP, Wouterlood F, eds), New York: Oxford UP.

Buzsaki G (1989) Two-stage model of memory trace formation: a role for "noisy" brain states. Neuroscience 31:551-570.

Buzsaki G (1996) The hippocampo-neocortical dialogue. Cereb Cortex 6:81-92.

de Curtis M, Pare D (2004) The rhinal cortices: a wall of inhibition between the neocortex and the hippocampus. Prog Neurobiol 74:101-110.

Ferino F, Thierry AM, Glowinski J (1987) Anatomical and electrophysiological evidence for a direct projection from Ammon's horn to the medial prefrontal cortex in the rat. Exp Brain Res 65:421-426.

Frankland PW, Bontempi B, Talton LE, Kaczmarek L, Silva AJ (2004) The involvement of the anterior cingulate cortex in remote contextual fear memory. Science 304:881-883.

Gilmartin MR, McEchron MD (2005) Single neurons in the medial prefrontal cortex of the rat exhibit tonic and phasic coding during trace fear conditioning. Behav Neurosci 119:1496-1510.

Hartigan JA, Hartigan PM (1985) The dip test of unimodality. Ann Statist 13:70-84.

Hurley KM, Herbert H, Moga MM, Saper CB (1991) Efferent projections of the infralimbic cortex of the rat. J Comp Neurol 308:249-276.

Jay TM, Witter MP (1991) Distribution of hippocampal CA1 and subicular efferents in the prefrontal cortex of the rat studied by means of anterograde transport of Phaseolus vulgaris-leucoagglutinin. J Comp Neurol 313:574-586.

Kim JJ, Fanselow MS (1992) Modality-specific retrograde amnesia of fear. Science 256:675-677.

Leutgeb S, Leutgeb JK, Moser MB, Moser EI (2005) Place cells, spatial maps and the population code for memory. Curr Opin Neurobiol 15:738-746.

Martina M, Royer S, Pare D (2001) Propagation of neocortical inputs in the perirhinal cortex. J Neurosci 21:2878-2888.

McClelland JL, McNaughton BL, O’Reilly RC (1995) Why there are complementary learning systems in the hippocampus and neocortex: insights from the successes and failures of connectionist models of learning and memory. Psychol Rev 102:419-457.

McEchron MD, Tseng W, Disterhoft JF (2003) Single neurons in CA1 hippocampus encode trace interval duration during trace heart rate (fear) conditioning in rabbit. J Neurosci 23:1535-1547.

McGaugh JL (2004) The amygdala modulates the consolidation of memories of emotionally arousing experiences. Annu Rev Neurosci 27:1-28.

Meunier M, Bachevalier J, Mishkin M, Murray EA (1993) Effects on visual recognition of combined and separate ablations of the entorhinal and perirhinal cortex in rhesus monkeys. J Neurosci 13:5418-5432.

Muir GM, Bilkey DK (2003) Theta- and movement velocity-related firing of hippocampal neurons is disrupted by lesions centered on the perirhinal cortex. Hippocampus 13:93-108.

Munera A, Gruart A, Munoz MD, Fernandez-Mas R, Delgado-Garcia JM (2001) Hippocampal pyramidal cell activity encodes conditioned stimulus predictive value during classical conditioning in alert cats. J Neurophysiol 86:2571-2582.

Murray EA, Graham KS, Gaffan D (2005) Perirhinal cortex and its neighbors in the medial temporal lobe: contributions to memory and perception. Q J Exp Psychol B 58:378-396.

Paz R, Pelletier JG, Bauer EP, Pare D (2006) Emotional enhancement of memory via amygdala-driven facilitation of rhinal interactions. Nat Neurosci 9:1321-1329.

Pelletier JG, Apergis J, Pare D (2004) Low-probability transmission of neocortical and entorhinal impulses through the perirhinal cortex. J Neurophysiol 91:2079-2089.

Pelletier JG, Apergis-Schoute J, Pare D (2005) Interaction between amyg- 
dala and neocortical inputs in the perirhinal cortex. J Neurophysiol 94:1837-1848.

Pinto A, Fuentes C, Pare D (2006) Feedforward inhibition regulates perirhinal transmission of neocortical inputs to the entorhinal cortex: ultrastructural study in guinea pigs. J Comp Neurol 495:722-734.

Room P, Russchen FT, Groenewegen HJ, Lohman AH (1985) Efferent connections of the prelimbic (area 32) and the infralimbic (area 25) cortices: an anterograde tracing study in the cat. J Comp Neurol 242:40-55.

Ryou JW, Cho SY, Kim HT (2001) Lesions of the entorhinal cortex impair acquisition of hippocampal-dependent trace conditioning. Neurobiol Learn Mem 75:121-127.

Savage LM, Buzzetti RA, Ramirez DR (2004) The effects of hippocampal lesions on learning, memory, and reward expectancies. Neurobiol Learn Mem 82:109-119.

Sesack SR, Deutch AY, Roth RH, Bunney BS (1989) Topographical organization of the efferent projections of the medial prefrontal cortex in the rat: an anterograde tract-tracing study with Phaseolus vulgaris leucoagglutinin. J Comp Neurol 290:213-242.

Shors TJ (2004) Memory traces of trace memories: neurogenesis, synaptogenesis and awareness. Trends Neurosci 27:250-256.

Sierra-Mercado Jr D, Corcoran KA, Lebron-Milad K, Quirk GJ (2006) Inactivation of the ventromedial prefrontal cortex reduces expression of conditioned fear and impairs subsequent recall of extinction. Eur J Neurosci 24:1751-1758.

Solomon PR, Vander Schaaf ER, Thompson RF, Weisz DJ (1986) Hippocampus and trace conditioning of the rabbit's classically conditioned nictitating membrane response. Behav Neurosci 100:729-744.

Squire LR, Alvarez P (1995) Retrograde amnesia and memory consolidation: a neurobiological perspective. Curr Opin Neurobiol 5:169-177.

Sutherland GR, McNaughton B (2000) Memory trace reactivation in hip- pocampal and neocortical neuronal ensembles. Curr Opin Neurobiol 10:180-186.

Suzuki WA, Eichenbaum H (2000) The neurophysiology of memory. Ann NY Acad Sci 911:175-191.

Swanson LW (1981) A direct projection from Ammon's horn to prefrontal cortex in the rat. Brain Res 217:150-154.

Takashima A, Petersson KM, Rutters F, Tendolkar I, Jensen O, Zwarts MJ, McNaughton BL, Fernandez G (2006) Declarative memory consolidation in humans: a prospective functional magnetic resonance imaging study. Proc Natl Acad Sci USA 103:756-761.

Takehara K, Kawahara S, Kirino Y (2003) Time-dependent reorganization of the brain components underlying memory retention in trace eyeblink conditioning. J Neurosci 23:9897-9905.

Takehara-Nishiuchi K, Nakao K, Kawahara S, Matsuki N, Kirino Y (2006) Systems consolidation requires postlearning activation of NMDA receptors in the medial prefrontal cortex in trace eyeblink conditioning. J Neurosci 26:5049-5058.

Thierry AM, Gioanni Y, Degenetais E, Glowinski J (2000) Hippocampoprefrontal cortex pathway: anatomical and electrophysiological characteristics. Hippocampus 10:411-419.

Vertes RP (2004) Differential projections of the infralimbic and prelimbic cortex in the rat. Synapse 51:32-58.

Weiss C, Kronforst-Collins MA, Disterhoft JF (1996) Activity of hippocampal pyramidal neurons during trace eyeblink conditioning. Hippocampus 6:192-209.

Wiltgen BJ, Brown RA, Talton LE, Silva AJ (2004) New circuits for old memories: the role of the neocortex in consolidation. Neuron 44:101-108.

Zola-Morgan S, Squire LR (1986) Memory impairment in monkeys following lesions limited to the hippocampus. Behav Neurosci 100:155-160. 